\title{
ACERCA DE LA ENSEÑANZA DE LA EXPRESIÓN ESCRITA DEL ESPAÑOL COMO LENGUA MATERNA EN LA ACTUALIDAD
}

\author{
María del Pilar Mesa Arroyo
}

\begin{abstract}
This contribution shows the current status of the study of the teaching of writing in the field of L1 Spanish teaching. With this aim, we first discuss the origin, topics of focus and the evolution of Applied Linguistics, and the teaching of language and literature. Next, we focus on the teaching of writing. This study first discusses different approaches to the teaching of this skill, before introducing the current dominant approach used in secondary education for the teaching of Spanish as a mother tongue: the communicative and functional approach. Finally, we formulate a proposal for the teaching of writing skills based on the microfunction analysis methodology, developed from the communicative and functional approach. The effectiveness of this methodology was the focus of previous studies, which are briefly discussed here.
\end{abstract}

Keywords: teaching; writing; L1 Spanish; Applied Linguistics; communicative approach.

Resumen: La presente contribución muestra el estado actual del estudio de la didáctica de la expresión escrita en el ámbito de la enseñanza del español como lengua materna. Para ello, en primer lugar, se hace un estudio del origen, objetos de atención y evolución de la Lingüística aplicada, y, seguidamente, de la Didáctica de la lengua y la literatura. A continuación, nos centramos en la didáctica de la expresión escrita, en los distintos enfoques existentes en la actualidad y en el enfoque predominante en la didáctica del español como lengua materna en la educación secundaria, el enfoque comunicativo y funcional. Por último, planteamos una propuesta de didáctica de la expresión escrita basada en la metodología del análisis microfuncional, que se inserta en este enfoque, y cuya efectividad ha sido demostrada en otros trabajos.

Palabras clave: didáctica; expresión escrita; español como lengua materna; Lingüística aplicada; enfoque comunicativo.

\section{Introducción}

En los últimos años los medios de comunicación, las evaluaciones externas, como el informe PISA ${ }^{1}$, y las internas, como las evaluaciones de diagnóstico llevadas a cabo por las distintas comunidades autónomas ${ }^{2}$, ponen de manifiesto una preocupación social por el nivel de instrucción de los estudiantes españoles en general, y en concreto, por la capacidad de expresión escrita de los alumnos de educación secundaria.

\footnotetext{
OCDE 2008.

2 Consejería de Educación de la Junta de Andalucía 2010; 2011.
} 
En la sociedad actual tiene una gran importancia ser capaz de expresarse con adecuación y corrección. Así uno de los objetivos que se plantea en nuestro sistema educativo para la etapa de la educación secundaria obligatoria es:

Comprender y expresar con corrección, oralmente y por escrito, en la lengua castellana y, si la hubiere, en la lengua cooficial de la Comunidad Autónoma, textos y mensajes complejos, e iniciarse en el conocimiento, la lectura y el estudio de la literatura. ${ }^{3}$

Del mismo modo, una de las competencias básicas que el alumno ha de desarrollar en esta etapa educativa es la competencia en comunicación lingüística. Esta tiene una gran relevancia para la socialización y la adquisición de nuevos conocimientos del alumnado.

Actualmente, a pesar de la expansión de los medios audiovisuales y del desarrollo de las tecnologías de la información y la comunicación, uno de los principales medios de comunicación, de difusión del conocimiento y de la información, sigue siendo el texto escrito. Además, es reconocido por todos que no es fácil desarrollar la capacidad de expresión escrita y que se necesita de una instrucción específica.

A pesar de ello, como profesora de Lengua Castellana y Literatura desde 2003 vengo observando las dificultades de los alumnos de la Educación Secundaria Obligatoria para componer textos adecuados a diferentes situaciones comunicativas. En lo que se refiere a la expresión escrita, una de las grandes deficiencias de este alumnado es la adecuación. Por una parte, este desconoce que en la composición de un texto en una situación formal, por ejemplo un control o un trabajo para entregar al profesor, se debe utilizar un registro formal, y por otra parte, aun siendo consciente de esta necesidad, el alumnado desconoce formas o expresiones adecuadas a este registro.

Asimismo, hemos comprobado, mediante un estudio realizado sobre libros de texto utilizados en la actualidad en la ESO para la materia de Lengua castellana y Literatura en Andalucía ${ }^{4}$, que estos libros de texto no incluyen actividades suficientes ni contribuyen adecuadamente al desarrollo de la capacidad de expresión escrita de este alumnado. ${ }^{5}$ Tampoco abundan las propuestas didácticas para la composición escrita en la ESO alternativas a estos manuales. Para primaria podemos citar a Querol Bataller (2011) y para secundaria a Mesa Arroyo (2011; 2012) y Gallego Bono (2009). Este último plantea una secuencia didáctica basada en los marcadores discursivos. Esta propuesta aboga por un enfoque mixto basado fundamentalmente en el enfoque por procesos y aplica una teoría lingüística actual, como es la de los marcadores discursivos, a la enseñanza del español como lengua materna, si bien, se basa exclusivamente en los marcadores discursivos.

Por todo lo anterior, llevamos a cabo una revisión bibliográfica de la didáctica de la expresión escrita en el ámbito de la enseñanza del español como lengua materna. En primer lugar, nos referimos al ámbito en el que se enmarcan las disciplinas que nos ocupan. En un sentido amplio la didáctica de la expresión escrita, tanto en la enseñanza de la lengua materna como extranjera, se inserta en el ámbito de lo que se conoce como Lingüística aplicada. Por consiguiente, nos referimos brevemente a las características, orígenes y objetos de estudio de esta disciplina. Seguidamente, nos detenemos en la consideración de la Didáctica de la lengua y la literatura.

\footnotetext{
3 Real Decreto 1631/2006, de 29 de diciembre, por el que se establecen las enseñanzas mínimas correspondientes a la Educación Secundaria Obligatoria.

4 Vid. Mesa Arroyo 2011.

5 A esta misma conclusión se llega en un estudio de libros de texto de primaria (Camps Pemán 2009).
} 
Posteriormente, nos ocupamos de la didáctica de la expresión escrita, y del enfoque predominante en la actualidad en la educación secundaria, el enfoque comunicativo y funcional. Por último, planteamos una propuesta de didáctica de la expresión escrita que se inserta en este enfoque basada en la metodología del análisis microfuncional.

El presente trabajo es relevante en tanto que son pocos los estudios que se dedican a la enseñanza del español como lengua materna en la actualidad, sobre todo, si los comparamos con aquellos que se ocupan del estudio del español como segunda lengua o lengua extranjera o de otros ámbitos de la Lingüística hispánica.

En lo que se refiere a la didáctica de la expresión escrita, si bien tradicionalmente se considera que se ha prestado mayor atención a esta didáctica que a la de la oralidad, no obstante, pensamos que es necesario prestar atención al estado actual de esta disciplina en la enseñanza del español como lengua materna debido a las deficiencias en este campo que presenta el alumnado español de educación secundaria y a la escasez de materiales didácticos adecuados para solventarlas.

\section{La Lingüística aplicada}

Se entiende esta disciplina como aplicación práctica de las teorías lingüísticas cuya finalidad es solucionar problemas y como punto de convergencia entre estas, y, en consecuencia, como campo interdisciplinar. En general, podemos afirmar que con la denominación de Lingüística aplicada se recogen las distintas posibles aplicaciones de las teorías lingüísticas (Marcos Marín, Sánchez Lobato 1991).

La propuesta de expresión escrita que presentamos en la última parte de este trabajo pertenece al ámbito de la Lingüística aplicada porque aplica una teoría lingüística, el concepto de microfunción y la metodología del análisis microfuncional, como propuesta para solventar un problema didáctico concreto: el hecho de que el alumnado de secundaria desconoce que ha de usar un registro formal en determinadas situaciones comunicativas y tipos textuales, y no dispone de medios gramaticales ni léxicos no coloquiales para utilizar en esas situaciones, mientras que la actual legislación educativa indica que el alumno ha de ser capaz al finalizar la etapa de la ESO de:

Utilizar la lengua para expresarse de forma coherente y adecuada en los diversos contextos de la actividad social y cultural y Emplear las diversas clases de escritos mediante los que se produce la comunicación con las instituciones públicas, privadas y de la vida laboral.

Como se ha dicho, una característica fundamental de la Lingüística Aplicada es la interdisciplinariedad ${ }^{6}$, así se considera en la definición de esta disciplina que se expone en Vez Jeremías procedente de Kaplan (1980: 57):

La LA es un conjunto de actividades relacionadas o de técnicas que median entre las distintas concepciones teóricas del lenguaje humano...; es el punto en el que todas las ramas de la lingüística llegan a converger (Vez Jeremías 1984: 15).

Por otra parte, la Lingüística Aplicada ha sido considerada, creemos que injustamente, como rama menor de la lingüística, en la que se ponían en práctica los principios de esta ciencia, y no como ciencia en sí misma (Marcos Marín, Sánchez Lobato: 1991).

Esta disciplina, tanto en un principio como en su desarrollo, se ha limitado frecuentemente al estudio de la enseñanza de segundas lenguas (González Nieto 2001: 30). En

\footnotetext{
6 Lacorte (2007: 34) apunta cinco rasgos esenciales de esta disciplina: perspectiva práctica, multidisciplinariedad, dimensión comunicativa, mediación teórico-práctica y conducta profesional.
} 
Marcos Marín y Sánchez Lobato (1991: 17-18) se señala que tiene sus orígenes en Norteamérica en la década de los cuarenta en el campo de la enseñanza de segundas lenguas «obviando otros aspectos que tienen cabida en su marco, y que reclaman interdisciplinariedad, como el aprendizaje de la lengua, la Psicolingüística, la Sociolingüística, la Lingüística computacional, etc.», pero, es, a partir de los años sesenta, cuando se define su objeto (Vez Jeremías 1984; Marcos Marín, Sánchez Lobato 1991).

Aparte de la atención dedicada a la enseñanza de lenguas extranjeras y a otros aspectos, queremos poner de manifiesto aquí la necesidad de que la Lingüística Aplicada se ocupe además de la enseñanza del español como lengua materna, como medio para la investigación encaminada a la resolución de problemas que surgen en este ámbito, ya que la investigación lingüística en el campo de la enseñanza del español como lengua materna no es todo lo frecuente que sería deseable. ${ }^{7}$ Ya en Peñalver Castillo (1991: 59) se manifestaba esta ausencia: «Hasta hace muy poco, pues, no se había hecho nada o casi nada por actualizar la enseñanza de la lengua materna y por incorporar a su metodología los hallazgos de la lingüística».

En la actualidad, Lacorte (2007) muestra en su publicación una visión sobre la Lingüística aplicada del español estructurada en tres campos a los que se dedica un número determinado de capítulos que detallamos a continuación:

- Aprendizaje y enseñanza del español: ${ }^{8} 6$ capítulos

- Entornos sociales, culturales y políticos: 5 capítulos

- El español como lengua profesional: 4 capítulos

Y en la que no se dedica ningún capítulo a la enseñanza del español como lengua materna.

En definitiva, en nuestra opinión es injusta la consideración de la Lingüística aplicada como rama menor de la Lingüística y abogamos por el desarrollo de propuestas en el ámbito objeto de nuestro estudio, la enseñanza del español como lengua materna, y en concreto, la didáctica de la expresión escrita en este ámbito con el fin de mejorar la competencia comunicativa del alumnado de educación secundaria.

A continuación, nos referimos a la disciplina que, dentro del campo de la Lingüística aplicada se ocupa la didáctica del español como lengua materna, y en la que se ubicaría la enseñanza de la expresión escrita en secundaria: la Didáctica de la lengua y la literatura.

\section{La enseñanza del español como lengua materna: la Didáctica de la lengua y la literatura}

De acuerdo con González Nieto (2001: 35) la Lingüística aplicada y la Didáctica de la lengua evolucionan convergentemente, lo cual quita importancia a las diferencias entre ambas que se caracterizan por la interdisciplinariedad. La aparición de la Didáctica de la lengua se produce por dos motivos: por la necesidad de solucionar problemas como «eficacia metodológica, dificultades específicas de la materia, deficiencias en el proceso de aprendizaje de los alumnos» (Mendoza y otros 1996: 406), y por otra parte, porque:

\footnotetext{
7 Si en la base de datos de tesis doctorales del Ministerio de Educación, que se puede consultar en www.educacion.es/teseo, introducimos el descriptor Lingüística Aplicada observamos que el porcentaje de tesis que se dedican a la enseñanza de la lengua materna en la actualidad es muy reducido.

8 Como segunda lengua.
} 
entra en crisis la concepción tradicional de la enseñanza lingüística, basada en los conocimientos analíticos y preceptivos de la gramática, y emergen en el panorama lingüístico, literario y psicopedagógico nuevas ciencias que ofrecen importantes aportaciones al campo de la enseñanza de la lengua y la literatura (Prado Aragonés 2004: 31).

Precisamente Prado Aragonés (2004: 34) señala que el objetivo de la Didáctica de la lengua y la literatura es elaborar propuestas ante problemas en la enseñanza de la lengua:

la mayoría de los especialistas coinciden en señalar como fin último de la misma intervenir en la identificación de los problemas relacionados con la enseñanza y aprendizaje de la lengua y la literatura, mediante la investigación en la acción; y en presentar propuestas válidas para solucionarlos, a partir del análisis de los condicionantes implicados en el proceso didáctico.

La propuesta didáctica que planteamos surge en respuesta a la deficiente adecuación en los escritos de nuestros alumnos, por la que aplicamos una metodología novedosa en el campo de la enseñanza en lengua materna como es el análisis microfuncional cuyo desarrollo no hubiese sido posible sin las aportaciones de las nuevas ciencias lingüísticas al campo de la enseñanza de la lengua.

En este sentido, estamos de acuerdo con Lomas (1993: 94) quien aboga por la aplicación didáctica de conceptos y teorías procedentes de nuevas tendencias de la lingüística en oposición a las ocupaciones tradicionales de la enseñanza de la lengua tales como las actividades gramaticales o las normas gráficas:

los asuntos relativos a los «actos de habla», a los aspectos no verbales de la comunicación, a las determinaciones socioculturales que regulan los intercambios comunicativos, a los procesos implicados en la comprensión y emisión de los mensajes o a los procedimientos lingüísticos y no lingüísticos, en definitiva, de creación del sentido, han permanecido ajenos con frecuencia a un trabajo escolar centrado, por el contrario, en las actividades gramaticales, en los usos escritos y en sus normas gráficas, en los modelos canónicos de la historia literaria.

El campo de la Didáctica de la lengua y la literatura tiene una historia relativamente breve, se remonta a los años 70 y no se constituye como área de conocimiento independiente en la universidad española hasta 1986, por ello, en este campo se dan abundantes fluctuaciones. Diversos trabajos han profundizado en la necesidad de delimitación y fundamentación de esta disciplina (Camps 1993; Mendoza, López Valero, Martos Núñez 1996). Se sitúa en la concurrencia de una diversidad de campos científicos como la Sociolingüística, la Sociología, la Lingüística, la Pragmática, la Pedagogía y la Metodología, la Psicolingüística y la Psicología, tal y como se puede observar en el gráfico 1 tomado de Camps (1998: 34) ${ }^{9}$. Desde luego, estas disciplinas no se amalgaman sin más, sino que realizan sus aportaciones con el fin de contribuir a la mejora de la enseñanza de la lengua.

En González Nieto (2001: 29) se indican los postulados generales de la Didáctica de la lengua y la literatura que podrían resumirse en la necesaria preeminencia de la enseñanza de las cuatro destrezas básicas: comprensión y expresión, orales y escritas:

a) La finalidad de la enseñanza de la lengua y de la literatura, en los niveles primario y secundario, es, ante todo, el desarrollo de las capacidades verbales de expresión y de comprensión.

\footnotetext{
9 Recientemente Lomas y Tusón (2012: 83) se congratulan de que en los últimos años haya un auge en las facultades de la pragmática, la lingüística del texto, el análisis del discurso o la sociolingüística, disciplinas que están orientadas a que los futuros docentes se centren en la mejora de la competencia comunicativa del alumnado.
} 
DISCIPLINAS RELACIONADAS CON LA ENSEÑANZA DE LA LENGUA

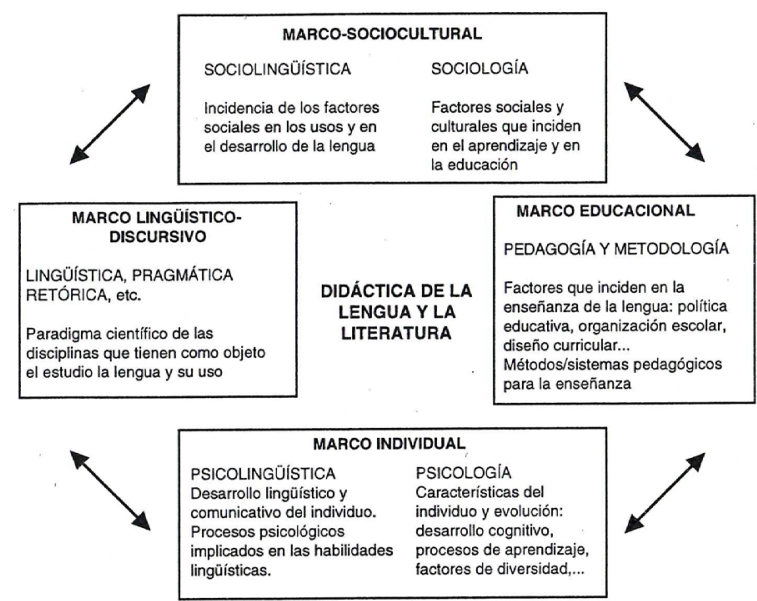

Gráfico 1 (tomado de Camps 1998: 34)

b) La actividad educativa debe dar preeminencia a la práctica, al uso de la lengua, a la lectura y a la expresión oral y escrita.

c) La actividad educativa también debe proporcionar conocimientos sobre la lengua, de carácter gramatical, siempre que estén relacionados con el uso y tengan, por lo tanto, un carácter práctico y normativo.

d) La enseñanza de la literatura no puede efectuarse al margen de la lectura y del comentario de los textos.

En la legislación educativa actual, tanto en los objetivos, como en los contenidos y en los criterios de evaluación, así como en el planteamiento del desarrollo de la competencia en comunicación lingüística, se observa este mismo enfoque comunicativo en el que lo que ha de predominar es la mejora de las destrezas básicas del alumno. ${ }^{10}$

Por último, en lo que se refiere al panorama actual de la disciplina, no podemos olvidarnos de los recientes avances metodológicos que suponen las llamadas TIC (tecnologías de la información y la comunicación) y TAC (tecnologías del aprendizaje y del conocimiento) ya que tal y como afirma Cassany (2011a: 1) «los usos de la lectura y la escritura del español han cambiado de manera drástica en los últimos años». Son numerosos los trabajos que se ocupan de la aplicación de las TIC en la didáctica de la lengua y la literatura, podemos citar a algunos entre muchos, como Alonso et al. (2010) y Vázquez Gutiérrez (2011); en lo que respecta a distintos tipos de actividades y recursos TIC, González Goñi (2011); en cuanto a Internet, Cassany (2011b) y el número 57 de la revista Textos $^{11}$, y acerca del uso de blogs, Zayas (2011).

\section{Didáctica de la expresión escrita}

De las cuatro destrezas objeto de la Didáctica de la lengua y la literatura, nos vamos a detener, en concreto, en una de ellas, la expresión escrita. Antes de exponer los enfoques

\footnotetext{
10 Asimismo, hace tiempo que los docentes de lengua y literatura dejaron de cuestionarse la orientación comunicativa de las enseñanzas lingüísticas (Lomas, Vera 2012: 7).

${ }^{11}$ Con un monográfico titulado Textos e hipertextos.
} 
y aportaciones más destacados dentro de este ámbito, es necesario señalar que la capacidad de expresión escrita ya no se considera un talento innato sino que es una destreza que se puede aprender y mejorar, y para ello, se necesita llevar a cabo una instrucción planificada (Monné 1998: 155).

Daniel Cassany (1990) resume los cuatro tipos de enfoques existentes en la enseñanza de la expresión escrita tanto de la lengua materna como de una lengua extranjera. Los cuatro enfoques didácticos que se exponen son:

1. Enfoque basado en la gramática

Nace en el contexto escolar de la enseñanza de la expresión escrita en la lengua materna, y luego se traspasa y adapta para la enseñanza de la escritura en L2. La idea básica es que para aprender a escribir se tiene que dominar la gramática de la lengua (las reglas que la construyen, la esencia, la estructura, la organización formal subyacente, etc.). El núcleo de la enseñanza lo constituye precisamente este conjunto de conocimientos gramaticales sobre la lengua: sintaxis, léxico, morfología, ortografía, etc., obviamente, la influencia más importante que recibe este enfoque proviene del campo de la lingüística o de la gramática.

\section{Enfoque basado en las funciones}

Nace en el contexto de la enseñanza de una L2 y, en concreto, en el seno de una metodología: la comunicativa. Sigue la tradición de métodos nocional-funcionales, desarrollados en Europa durante los años sesenta, en los que lo más importante es enseñar una lengua para usarla, para comunicarse. Este tipo de métodos tiene su origen en la filosofía del lenguaje (Wittgenstein, Austin, Searle, etc.), y en la concepción funcionalista de la lengua que desarrolló ésta. También recibe influencias de la sociolingüística, de los primeros trabajos sobre lingüística del texto y, en el campo de la didáctica, de los movimientos de renovación pedagógica y de enseñanza activa.

\section{Enfoque basado en el proceso}

A partir de los años setenta se desarrolló en Estados Unidos un conjunto de investigaciones sobre el proceso de producción o composición de textos escritos [...] Estas investigaciones y el enfoque general en la didáctica de la expresión escrita reciben mucha influencia de la psicología cognitiva.

En la actualidad este modelo cognitivo, según el cual hay que incidir en el proceso de la composición, es la tendencia fundamental en la que se basan las propuestas de actividades de expresión escrita.

A nivel internacional desarrolla este modelo Flower (1985) y en nuestro entorno lo hacen Camps y Cassany (1987). En esta línea se inserta la propuesta que elaboran Gómez Rodríguez y Fernández Rodríguez (2009: 351 y 352).

4. Enfoque basado en el contenido

Este enfoque se desarrolló en Estados Unidos durante la década de los ochenta [...] la idea fundamental que subyace es la supremacía del contenido por encima de la forma (sea ésta gramática, función, tipo de texto o proceso) [...] Otras experiencias didácticas muy relacionadas con este enfoque basado en el contenido son el trabajo organizado por tareas.

Cortés (2000: 268) habla de la enseñanza de la lengua mediante tareas (Task-Based Language Teaching) también conocida como enfoque por tareas (Task-Based Approach) como modelo didáctico engendrado en el seno de la enseñanza comunicativa de la lengua en un afán de superación de este. En él se da prioridad al aprendizaje social y cooperativo, mediante la realización de tareas de comunicación real que fomenten y desarrollen la plena competencia comunicativa del alumno. 
Otro método es el trabajo por proyectos definido por Camps (2003: 47) de este modo:

El punto de partida de todos los proyectos a los que se hace referencia es que la lengua además de ser objeto de aprendizaje es instrumento para llevar a cabo actividades diversas. En algunos casos, la lengua es primordialmente el instrumento para la elaboración, recopilación y organización de los conocimientos y para comunicarlos, oralmente y por escrito.

Como hemos visto, Cassany (1990) clasifica los enfoques de la expresión escrita, y, finalmente, propone el eclecticismo. Coincidimos con este autor en la necesidad de no decantarse por uno solo de estos enfoques, creemos que un enfoque funcional puede atender a aspectos gramaticales y léxicos, y al contenido y, enclavarse en un enfoque procesual. Defendemos el planteamiento de actividades verosímiles en contextos comunicativos determinados que permitan al alumno obtener medios léxicos y gramaticales para la expresión de funciones comunicativas y semántico-gramáticales, teniendo en cuenta el contenido que quieren expresar. El enfoque que proponemos se centra fundamentalmente en el análisis microfuncional, si atendemos a la clasificación de enfoques propuesta por Cassany (1990), no descuida la atención al contenido ni a la gramática ${ }^{12}$. En lo que se refiere al enfoque basado en el proceso, nuestra propuesta de análisis microfuncional puede insertarse en un enfoque procesual estructurándose la tarea propuesta en tres etapas: planificación, textualización y revisión.

\section{Enfoque comunicativo y funcional}

En cuanto a la terminología, Cortés considera sinónimos enfoque comunicativo, enseñanza comunicativa de la lengua, enfoque nocional-funcional o enfoque funcional (Cortés 2000: 264). Por ello, vamos a utilizar indistintamente estos términos para referirnos a este enfoque, que Cassany denomina enfoque por funciones, y que se caracteriza por dar preeminencia a lo que se desea comunicar o a la función comunicativa que se realiza.

Prado Aragonés señala que el enfoque comunicativo y funcional se basa en «el uso adecuado de la lengua en situaciones de comunicación concreta» (Prado Aragonés 2004: 258) y que surge en los años setenta derivado de los avances de las ciencias del lenguaje y la psicología. Mediante este enfoque se pretende que los alumnos adquieran una adecuada competencia comunicativa. Para Vez Jeremías (1998: 84) el enfoque comunicativo: «Centra la enseñanza-aprendizaje de lenguas en un proceso como comunicación y para la comunicación, sobrepasando el ámbito oracional para abarcar el ámbito discursivo».

Lo más importante de este enfoque es el énfasis en la comunicación o en el uso de la lengua, contraponiéndolo al enfoque gramatical anterior, en el que lo importante era la estructura de la lengua, las reglas de gramática (Cassany 1990).

Cassany (1990) traza las líneas teóricas y presenta la práctica de este enfoque. De acuerdo con este autor, los contenidos y objetivos de un curso que siga este enfoque son el conjunto de actos de habla y los recursos lingüísticos correspondientes. El contenido de la clase son los mismos usos de la lengua, tal como se producen en la calle (y no la gramática abstracta que les subyace). La metodología es muy práctica, el alumno está constantemente activo en el aula: escucha, lee, habla con los compañeros, practica, etc.

Es interesante el tardío desarrollo de este enfoque en la didáctica de la expresión escrita y la importancia que los tipos de textos tienen en este. Cassany (1990) señala que en sus

${ }^{12}$ La necesidad de relacionar la expresión escrita con conocimientos sobre la lengua y la norma la concreta el Real Decreto en el objetivo número once de la materia: «Aplicar con cierta autonomía los conocimientos sobre la lengua y las normas del uso lingüístico para comprender textos orales y escritos y para escribir y hablar con adecuación, coherencia, cohesión y corrección». 
inicios este enfoque se desarrolló en la enseñanza del inglés y del francés como lengua extranjera para estudiantes de primeros niveles, de ahí que apenas se prestara atención a la expresión escrita. No es hasta principios de los años ochenta, cuando empiezan a desarrollarse métodos de expresión escrita con estos planteamientos. En ellos se incorporan algunos de los hallazgos más importantes de la Lingüística del texto, como son los conceptos sobre las propiedades del texto (coherencia, cohesión, adecuación, etc.), o las tipologías de textos.

Resumimos las características de este enfoque ${ }^{13}$ siguiendo, de nuevo, a Cassany (1990):

- Visión descriptiva de la lengua, opuesta a la visión prescriptiva anterior. Se enseña la lengua tal como la usan los hablantes. Se sustituye el binomio correcto / incorrecto por el de adecuado / inadecuado.

-Varios modelos lingüísticos: dialectos y registros. Un curso de lengua debe ofrecer modelos lingüísticos variados: un alumno debe poder entender varios dialectos de la misma lengua y, también, dentro del estándar que tiene que dominar productivamente, ha de poder utilizar un registro formal y otro más coloquial.

- Materiales ¿reales o realistas? Los textos que se utilizan para la clase deben ser reales o, como mínimo, verosímiles.

- Atención especial a las necesidades comunicativas de cada alumno. Cada alumno tiene necesidades comunicativas distintas, de modo que deberá aprender funciones y recursos lingüísticos distintos.

- En los métodos nocional-funcionales, la programación se basa en un conjunto de funciones o actos de habla. En los métodos exclusivos de lengua escrita, la programación se basa en la tipología de textos desarrollada por la lingüística del texto.

- En una clase se actúa de la siguiente forma: 1. Se presentan varios ejemplos reales o verosímiles de un determinado tipo de texto, 2. Se analizan los modelos, se comparan entre sí para extraer las características generales del tipo de texto: partes de la estructura, estilo y fraseología; tipo de información que incluye, etc., 3. Prácticas cerradas de producción escrita; los alumnos trabajan con textos preparados en tareas preparatorias y parciales, 4 . Prácticas comunicativas: dado un contexto o una situación determinada, los alumnos escriben un texto completo, 5 . El profesor corrige los trabajos.

Esta metodología ha sido utilizada fundamentalmente en la enseñanza de lenguas extranjeras y en los estudios de traducción. Los ejemplos de este enfoque para el español que cita Cassany son métodos de español como lengua extranjera. Sin embargo, consideramos que el enfoque funcional ya aplicado en lenguas extranjeras debe ser el que se aplique en la enseñanza en la lengua materna. Algunos autores han apuntado esta misma necesidad (González Nieto 2001: 13).

La propuesta didáctica de composición que formulamos en el apartado siguiente, basada en microfunciones, se inserta fundamentalmente en el enfoque comunicativo ya que propone la composición en una situación de comunicación realista formal con textos verosímiles y el alumno se basa en lo que ha de expresar para seleccionar los recursos lingüísticos que necesita, además ha de adecuarse a un destinatario y a las convenciones de un tipo textual concreto.

La finalidad de la enseñanza de la lengua en el sistema educativo actual es desarrollar la competencia comunicativa del alumnado. En el Real Decreto 1631/2006, de 29 de diciembre,

${ }^{13}$ En Mendoza, López y Martos $(1996: 73,74)$ se exponen también las características de este enfoque. 
por el que se establecen las enseñanzas mínimas correspondientes a la Educación Secundaria Obligatoria se indica que el objetivo de la materia de Lengua Castellana y Literatura es el desarrollo de la competencia comunicativa, es decir, un conjunto de conocimientos sobre la lengua y de procedimientos de uso que son necesarios para interactuar satisfactoriamente en diferentes ámbitos sociales. Del mismo modo, de acuerdo con la definición de competencia en comunicación lingüística que contiene el citado Real Decreto «el desarrollo de la competencia lingüística al final de la educación obligatoria comporta el dominio de la lengua oral y escrita en múltiples contextos». Así mismo, el concepto de competencia comunicativa se considera el origen del giro constatado en la concepción de la enseñanzaaprendizaje de lenguas en los currículos desarrollados a partir de la LOGSE y es el propósito fundamental de un enfoque funcional comunicativo y de nuestra propuesta.

Como vemos en la enseñanza del español como lengua materna en la ESO, las disposiciones legales de nuestro actual sistema educativo siguen un enfoque eminentemente comunicativo, en el cual se proponen como objetivos fundamentales que el alumno comprenda y se exprese adecuadamente tanto de forma oral como escrita y se da una importancia fundamental a la construcción de textos en función de determinadas finalidades y con propósitos diversos, en este sentido, una perspectiva funcional se integra perfectamente en el currículo actual. Esta perspectiva la encontramos, por ejemplo, en el temario de oposiciones para profesores de Educación secundaria de Lengua Castellana y Literatura en el cual aparecen cinco temas con este enfoque ${ }^{14}$.

Con este enfoque comunicativo y funcional se desarrollan el concepto de microfunción y de análisis microfuncional, de los que nos ocupamos a continuación, y que han sido aplicados con éxito en el ámbito de la traducción. Este tipo de análisis se desarrolló en el proyecto de investigación I+D Gramática funcional contrastiva (español-alemán) para traductores y/o intérpretes (enfoque onomasiológico) [PB-98 1336 $]^{15}$, cuyo objetivo era la elaboración de una gramática funcional contrastiva de español-alemán para traductores con enfoque onomasiológico. Los resultados de este proyecto han despertado interés a nivel internacional (Vilar 2001). En el ámbito de la enseñanza de la lengua materna, se ha demostrado la efectividad de la aplicación de esta metodología en el alumnado de Lengua castellana y literatura de la $\mathrm{ESO}^{16}$.

\section{Didáctica de la expresión escrita basada en el análisis microfuncional}

El término función en lingüística se ha utilizado con múltiples sentidos, tal y como lo emplearon distintos autores como Roman Jakobson, André Martinet, la Escuela de Praga, Emilio Alarcos Llorach, Michael Halliday, Hjemslev o S. Dik. En la enseñanza de idiomas generalmente el término función se usa para referirse a los diferentes actos sociales que un emisor puede llevar a cabo en la lengua extranjera: presentarse, pedir permiso, ofrecer, etc. En el ámbito de la enseñanza de lenguas extranjeras se habla con frecuencia de función comunicativa aludiendo a los usos y fines del lenguaje dentro de la comunicación humana recogidos en forma de repertorios con el fin de construir sílabos o programas didácticos. A veces se habla de enfoque nocional-funcional con lo que se hace referencia

\footnotetext{
14 18.- Elementos lingüísticos para la expresión de la cantidad, la cualidad y el grado. 19.- Elementos lingüísticos para la expresión del tiempo, el espacio y el modo. 20.- Expresión de la aserción, la objeción, la opinión, el deseo y la exhortación. 21.- Expresión de la duda, la hipótesis y el contraste. 22.- Expresión de la causa, la consecuencia y la finalidad.

15 Desarrollado durante los años 2000, 2001 y 2002.

16 Vid. Mesa Arroyo 2011; 2012.
} 
a nociones como tiempo, espacio o lugar. Los enfoques nociofuncionales basados en estas funciones comunicativas se desarrollan a partir de los años setenta en el ámbito de los proyectos de difusión de lenguas extranjeras promovidos por el Consejo de Europa.

El concepto de microfunción tiene su origen en el de función comunicativa, al que nos acabamos de referir y en el de acto de habla. El concepto de microfunción, fundamento de nuestra propuesta didáctica, tiene su base en el de acto de habla. Se especifica en Vilar (2008: 149) que: «Frecuentemente, las microfunciones coinciden con los actos ilocucionarios descritos por Austin (1962) y Searle (1969), pero en muchos casos los actos ilocucionarios son más complejos y se componen de varias microfunciones».

La metodología del análisis microfuncional parte del análisis del texto como unidad lingüística y su finalidad es la composición mediante el enfoque funcional comunicativo. Esta metodología a partir de un enfoque onomasiológico permite la adecuación a diferentes tipos de textos, situaciones comunicativas, destinatarios y propósitos comunicativos, y ayuda al alumno a la mejora de la adecuación estilística, y, por tanto, de su competencia comunicativa. En contraposición al enfoque semasiológico, que es aquel que se ha utilizado tradicionalmente en diccionarios y gramáticas, en el que partiendo de la forma lingüística se llega al significado. El enfoque onomasiológico va desde el significado a la forma y ha tenido un importante desarrollo en la enseñanza de segundas lenguas, en cuyos manuales y gramáticas, cada vez con más frecuencia, se recogen diferentes funciones comunicativas con las formas que permiten su expresión ${ }^{17}$.

Las microfunciones son definidas por Vilar (2004: 304) como las «unidades comunicativas, semántico-gramaticales y pragmáticas mínimas del texto». El análisis microfuncional, introducido por Vilar, es una metodología de análisis textual que parte de las microfunciones. El objetivo de este análisis es la descripción del repertorio y del uso de formas y estructuras lingüísticas específicas en función del tipo de texto en el que aparecen, es decir, la descripción estilística de diferentes tipos de texto.

El análisis microfuncional siempre se aplica en función de un determinado tipo de texto porque la finalidad consiste en describir el uso específico de los recursos lingüísticos en función de ese criterio, ya que los distintos tipos textuales tienen composiciones funcionales diferentes. Es decir, la finalidad de esta metodología es describir un estilo determinado en cada caso.

Esta propuesta se inserta en el enfoque funcional comunicativo y en las disposiciones del actual sistema educativo puesto que se plantea al alumno la creación de un tipo textual en una situación verosímil en el que ha de utilizar un registro formal. Mediante la metodología que proponemos, el alumno obtiene información de las funciones comunicativas y semántico-gramaticales y de los medios léxicos y gramaticales concretos (que denominamos con una terminología accesible), que se utilizan en ese tipo de texto. Con ello, el alumno conoce las microfunciones que ha de expresar $\mathrm{y}$, a partir de ellas, elige uno de los medios adecuados de los que ha obtenido mediante el análisis. De este modo, el propio alumno habrá elaborado con anterioridad a la propia composición de su texto, un pequeño repertorio de recursos contextualizados y adecuados estilísticamente. Esta será su herramienta de trabajo, que lo orientará en el uso de los recursos lingüísticos.

\footnotetext{
17 Vid. la Gramática comunicativa del español de Matte Bon (1992), asimismo en Mesa Arroyo (2008) recogemos un número de gramáticas o manuales de enseñanza del español como segunda lengua que al menos en una parte están estructuradas de acuerdo con un enfoque onomasiológico.
} 
Es posible poner en práctica la propuesta que planteamos utilizando las TIC como recurso didáctico. En el caso de Andalucía, actualmente los centros de secundaria cuentan, como mínimo, con pizarras digitales en todas las aulas de $1^{\circ}$ y $2^{\circ}$ de ESO, todo el alumnado de $1^{\circ}, 2^{\circ}$ y $3^{\circ}$ de esta etapa dispone de ordenadores portátiles propios, y en los institutos suele haber, al menos, un proyector y una sala con ordenadores. Aprovechando estos recursos, los/las alumnos/as, con el asesoramiento del profesor o profesora, buscarán en la red los modelos textuales para su análisis. El/la profesor/a, utilizando la pizarra digital o proyector, presentará la composición microfuncional de los modelos textuales mediante el programa Atlas.ti ${ }^{18}$ que permite mostrar los recursos lingüísticos de un modo fácil e intuitivo. Los/las alumnos/as tomarán nota de los resultados del análisis en tablas elaboradas por ellos mismos y a partir de estos elaborarán sus composiciones utilizando el procesador de textos, posteriormente, se ayudarán del corrector ortográfico para revisarlas. Por último, mediante correo electrónico enviarán al docente sus textos para su evaluación.

En el ámbito de la enseñanza de la lengua materna en secundaria González Nieto (2001: 304-305) ha señalado la pertinencia de un enfoque que enseñe al alumno las distintas formas con las que se puede expresar una misma función comunicativa o semánticogramatical:

Si la expresión de las relaciones lógico-sintácticas, como la causa, es realizable mediante formas diferentes [...] La formulación de un problema, ¿cómo expresar la causa?, y la comparación de enunciados como éstos puede y debe dar lugar a transformaciones acompañadas de una reflexión sobre las ventajas y desventajas de unos y otros -intención, registro... - en diferentes contextos.

En Mesa Arroyo $(2011 ; 2012)$ se ha demostrado la efectividad de la aplicación de la metodología del análisis microfuncional en 121 alumnos/as de los cuatro cursos de la ESO durante tres cursos escolares (2006/2007, 2007/2008 y 2008/2009). En todos los grupos la mayoría de los alumnos mejoró la composición de sus textos, tras aplicar esta metodología, estando el porcentaje de alumnos que mejora siempre por encima del $50 \%{ }^{19}$. Los textos elaborados tras aplicar la metodología propuesta resultaron más adecuados que los elaborados en primer lugar por el alumnado ya que respetaban en mayor medida las convenciones en cuanto a la presentación y a la superestructura; eran mejores en la selección de información apropiada; eran más adecuados estilísticamente al tipo de texto, al propósito comunicativo, al interlocutor; y presentaban un registro más formal.

\section{Conclusiones}

En este trabajo se ha dado una visión del estado actual de la didáctica de la expresión escrita como lengua materna. En primer lugar, se ha ubicado esta disciplina en la más amplia Lingüística aplicada destacando la necesidad de no considerarla como rama menor de la Lingüística y del desarrollo de propuestas en el campo de la enseñanza del

\footnotetext{
18 Atlas.ti es un CAQDAS (Computer Assisted Qualitative Data Analysis Software), los CAQDAS son programas pensados para facilitar el trabajo en el ámbito de las ciencias sociales, haciendo posible el procesamiento de grandes volúmenes de datos textuales, facilitando su análisis cuantitativo y cualitativo. Puede obtenerse una versión gratuita de demostración del programa en http//:www.atlas.ti.de. La versión es plenamente funcional, aunque con limitaciones en cuanto a la cantidad de datos analizables.

19 Tanto los modelos textuales entregados al alumnado como todos los textos compuestos por este antes y después de realizar el análisis microfuncional fueron presentados a través de la herramienta informática Atlas. ti que permite presentar de una manera ordenada y dinámica los textos, su composición microfuncional, las inadecuaciones observadas en estos, así como los criterios de evaluación aplicados.
} 
español como lengua materna con el fin de solventar las deficiencias en la competencia comunicativa de este alumnado, especialmente en la etapa secundaria.

Del mismo modo, se ha recogido el estado actual de la Didáctica de la lengua y la literatura, cuyo objetivo es la resolución de problemas metodológicos respecto a las deficiencias en los procesos de aprendizaje del alumnado y a las dificultades de la materia y que se sitúa en la concurrencia de disciplinas como la Sociolingüística, la Pragmática, la Pedagogía y la Psicolingüística. Además, se ha hecho referencia a la importancia de las TIC en esta disciplina en la actualidad.

Seguidamente, nos hemos centrado en la didáctica de la expresión escrita resumiendo los principales enfoques actuales: enfoques basados en la gramática, en las funciones, en el proceso, en el contenido; enfoque por tareas y trabajo por proyectos. Posteriormente, nos hemos detenido en el enfoque comunicativo y funcional trazando sus líneas teóricas y resumiendo sus características. Respecto a este se ha destacado que si bien esta metodología ha sido utilizada fundamentalmente en la enseñanza de lenguas extranjeras y en los estudios de traducción, en la enseñanza del español como lengua materna en la ESO, las disposiciones legales del actual sistema educativo siguen un enfoque eminentemente comunicativo.

Por último, se ha planteado el concepto de microfunción y la metodología del análisis microfuncional cuya efectividad ha sido demostrada recientemente en la didáctica de la expresión escrita del español como lengua materna.

\section{Bibliografía}

Alonso, Cristina - Casablancas, Silvina - Domingo, Laura - Guitert, Montserrat - Moltó, Óscar - SÁnchez, Joan-Anton, et al. (2010), «De las propuestas de la administración a las prácticas del aula», Revista de Educación 352, 53-76.

CAMPS, Ana (1993), «Didáctica de la lengua: la emergencia de un campo científico específico», Infancia y Aprendizaje 62-63, 209, 217.

CAMPS, Ana (1998), «La especificidad del área de la Didáctica de la lengua. Una visión sobre la delimitación de los contenidos de la enseñanza de la lengua y la literatura», in: Mendoza Fillola, Antonio: Conceptos clave en didáctica de la lengua y la literatura, Barcelona: ICE Horsori, 33-47.

Camps Pemán, Carmen (2009), El enfoque comunicativo en los libros de texto de Lengua Castellana para Educación Primaria. Análisis de los materiales utilizados en la Comunidad Autónoma del País Vasco, San Sebastián. Tesis doctoral que se puede consultar en https: / / www.educacion.es/ teseo/ mostrarRef.do?ref=835509.

CASSANY, Daniel (1987), Describir el escribir, Barcelona: Paidós.

CASSANY, Daniel (1990), «Enfoques didácticos para la enseñanza de la expresión escrita», Comunicación, lenguaje y educación 6: 63-80. http://www.upf.edu/dtf/ personal/danielcass/enfoques.htm.

CAssany, Daniel (2011a), «Después de Internet...», Textos de Didáctica de la Lengua y de la Literatura 57, 12-22.

CASSANY, Daniel (2011b), «El español escrito en contextos contemporáneos», Cuadernos Comillas 1, 1-4. http://www.cuadernoscomillas.es/pdf/1_presentacion_cassany. pdf.

Consejería de Educación de la Junta de Andalucía (2010), Evaluación de diagnóstico curso 2008-2009, Junta de Andalucía. (http://www.juntadeandalucia.es/ educacion/portal/com/bin/Contenidos/OEE/evaluacion/Evaluaciondiag 
nostico0809/1246617499991_evaluacixndiagnxstico2008_09.pdf, fecha última consulta: 9 de febrero de 2012).

Consejería de Educación de la Junta de Andalucía (2011), Evaluación de diagnóstico 2009-2010. Avance, Agencia Andaluza de Evaluación Educativa.

Cortés Moreno, Maximiano (2000), Guía para el profesor de idiomas, Barcelona: Octaedro.

FLOWER, Linda (1985), Problem-Solving Strategies for Writing, Brace Jovanovich (2a ed.).

Gallego Bono, Mercedes (2009), Marcadores discursivos en el proceso de composición del texto escrito. Propuesta didáctica, Valencia: Servei de Publicacions de la Universitat de Valencia. (http:/ / www.tesisenred.net/handle/10803/10326).

Gómez Rodríguez, José María - Fernández Rodríguez, Juan José (2009), «La competencia en comunicación lingüística», in: MONTORO DEL ARCO, Esteban Tomás (ed.) (2009), El español del siglo XXI. Actas de las XIV Jornadas sobre la lengua española y su enseñanza, Granada: Universidad de Granada, 341-356.

GONZÁLEZ GoÑI, Silvia (2011): «Actividades TIC en el aula: la innovación en pequeñas dosis», in: Hernández Ortega, José - Pennesi Fruscio, Máximo - Sobrino López, Diego - VÁzQuez Gutiérrez, Azucena (eds.), Experiencias educativas en las aulas del s. XXI, Barcelona: Ariel y Fundación Telefónica, 118-120.

GonZÁlez Nieto, Luis (2001), Teoría lingüística y enseñanza de la lengua, Madrid: Cátedra.

KaPLAN, Robert (ed.) (1980), On the scope of applied linguistics, New York: Newbury House.

LACORTE, Manel (coord.)(2007), Lingüística aplicada del español, Madrid: Arco Libros.

Lomas, Carlos - Osoro, Andrés (comps.) (1993), El enfoque comunicativo de la enseñanza de la lengua, Barcelona: Paidós.

Lomas, Carlos - Osoro, Andrés - Vera, Manuel (2012), «Bibliotecas escolares y planes de lectura», Textos de Didáctica de la Lengua y de la Literatura 60, 7-12.

LOMAs, Carlos - Osoro, Andrés - Tusón, Amparo (2012), «Lengua, cultura y sociedad. Una mirada sociolingüística a la enseñanza de la lengua», Textos de Didáctica de la Lengua y de la Literatura 60, 77-91.

Marcos Marín, Francisco - SÁnchez Lobato, Jesús (1991), Lingüística aplicada, Madrid: Síntesis.

Matte Bon, Francisco (1992), Gramática comunicativa del español, Madrid: Edelsa.

Mendoza Fillola, Antonio - López Valero, Amando - Martos Núñez, Eloy (1996), Didáctica de la lengua para la enseñanza primaria y secundaria, Madrid: Akal.

Mesa Arroyo, María del Pilar (2008), «Un acercamiento a las gramáticas de español como lengua extranjera», redELE 12. (http://www.educacion.es/redele/ Revista12/PilarMesa.pdf).

Mesa Arroyo, María del Pilar (2011), Propuesta de composición textual basada en «microfunciones» en la enseñanza del español como lengua materna en la ESO, Granada: Universidad de Granada (http://Ohera.ugr.es.adrastea.ugr.es/ tesisugr/19655782.pdf).

Mesa Arroyo, María del Pilar (2012), «Propuesta didáctica de composición textual con ayuda de microfunciones en la educación secundaria obligatoria», Textos de Didáctica de la Lengua y de la Literatura 60, 102-107. 
Monné Marsellés, Pilar (1998), «La escritura y su aprendizaje», in: Mendoza Fillola, Antonio: Conceptos clave en didáctica de la lengua y la literatura, Barcelona: ICE Horsori, 155-168.

Peñalver Castillo, Manuel (1991), La lingüística y la enseñanza de la lengua española en el bachillerato, Granada: Comares.

Prado Aragonés, Josefina (2004), Didáctica de la lengua y la literatura para educar en el siglo XXI, Madrid: La Muralla.

Querol Bataller, María (2011), «Enseñanza de la lengua escrita. Una experiencia con las 〈letras〉 chinas», Profesorado. Revista de currículum y formación del profesorado vol. 15, n 3, 313-327.

«Real Decreto 1631/2006, de 29 de diciembre, por el que se establecen las enseñanzas mínimas correspondientes a la Educación Secundaria Obligatoria», BOE, núm. 5 (5 enero 2007).

VÁzQuez Gutiérrez, María Azucena (2011), «Contenidos digitales en el aula», in: Hernández Ortega, José - Pennesi Fruscio, Máximo - Sobrino López, Diego VÁzquez Gutiérrez, Azucena (eds.), Experiencias educativas en las aulas del s. XXI, Barcelona: Ariel y Fundación Telefónica, 339-343.

Vez JeremíAs, José Manuel (1984), Claves para la lingüística aplicada, Málaga: Ágora.

Vez Jeremías, José Manuel (1998), «Enseñanza y aprendizaje de las lenguas», in: Mendoza Fillola, Antonio, Conceptos clave en didáctica de la lengua y la literatura, Barcelona: ICE Horsori, 75-86.

Vilar SÁncheZ, Karin (2001), «How Functionally Organized Grammars Could Help Learners Learn German Better?», Die Unterrichtspraxis-Teaching German 34.2, 169-177.

Vilar SÁnchez, Karin (2004), «Diccionario de sinónimos funcionales» in: Faber, Pamela - Jiménez Hurtado, Catalina - Wotjak, Gerd (Hrsg.), Léxico especializado y comunicación interlingüística, Granada: Universidad de Granada y Universidad de Leipzig, Serie Granada Lingvistica, 297-322.

VILAR SÁNCHEZ, Karin (2008), «El análisis de textos informatizados en la enseñanza de lenguas», in: Linde López, Ángeles - SANTAMARía LaRio, Juana - WallHEAD Galway, Celia Margaret (coords.) Studies in honour of Neil McLaren: a man for all seasons, Granada: Universidad de Granada, 145-158.

Zayas, Felipe (2011), «Dos usos del blog de aula», in: Hernández Ortega, José Pennesi Fruscio, Máximo - Sobrino LóPez, Diego - VÁzquez Gutiérrez, Azucena (eds.), Experiencias educativas en las aulas del s. XXI, Barcelona: Ariel y Fundación Telefónica, 153-156.

María del Pilar Mesa Arroyo
IES Alfaguara
C/Hoyo de Narváez, s/n,
18300 Loja
Granada
España
pilarmesa@yahoo.es

